\title{
INTERVAL REPRESENTATIONS OF CLIQUES AND OF SUBSET INTERSECTION GRAPHS
}

\author{
Edward R. Scheinerman ${ }^{1}$ \\ Johns Hopkins University \\ Baltimore, Maryland \\ Douglas B. West ${ }^{2}$ \\ University of Illinois \\ Urbana, Illinois 61801
}

\begin{abstract}
Constructions and bounds are given for the largest clique that can be represented as an intersection graph of intervals on the real line with bounded depth and multiplicity. Also, for the graph defined on the subsets of a set by putting vertices adjacent if and only if the corresponding subsets intersect, the interval number and boxicity are examined.
\end{abstract}

Keywords: intersection graph, interval number, boxicity

\section{Introduction}

In this note, we consider the use of intervals to represent two classes of highly symmetric graphs, in fact with $n$ !-fold symmetry. The first are the complete graphs. The second are the graphs whose vertices correspond to the subsets of an $n$-set, with vertices adjacent if and only if the corresponding sets intersect. The symmetry is important in each discussion. First we define the parameters to be studied.

Consider representing a graph by assigning each vertex $v$ a subset $f(v)$ of the real line, such that vertices are adjacent if and only if the corresponding subsets intersect. If each vertex is assigned a set consisting of at most $t$ intervals, we have a $t$-interval representation. The interval number $i(G)$ of a graph $G$ is the minimum $t$ such that $G$ has a $t$-interval representation. The graphs with interval number 1 are called interval graphs, and have been thoroughly studied and characterized. If each point of the line appears in sets assigned to at most $r$ vertices of $G$, the representation has depth $r$. The depth $r$ interval number $i_{r}(G)$ is the minimum $t$ such that $G$ has a $t$-interval representation of depth $r$. Letting $\Delta(G)$ denote the maximum vertex degree in $G$ and $\omega(G)$ the maximum clique size, note that $i(G)=i_{\omega}(G) \leq \cdots \leq i_{2}(G) \leq \Delta(G)$, so all these parameters are well defined. In fact, Griggs and West [1] showed $i_{2}(G) \leq\lceil(\Delta(G)+1) / 2\rceil$. In Section 2, we show $\left\lceil 1 / 2\left(\frac{n-1}{r-1}+\frac{r}{n}\right)\right\rceil \leq i_{r}\left(K_{n}\right) \leq 1 / 2 \frac{n}{r-1}+2\left\lceil\log _{r} n\right\rceil+1$. In other words, the largest clique representable by intervals with depth at most $r$ and multiplicity at most $t$ has about $2 t(r-1)$ vertices.

\footnotetext{
$\overline{1}$ Research supported in part by (numbers to be supplied)

${ }^{2}$ Research supported in part by an Arnold O. Beckman Fellowship, ONR Grant N00014-85K0570, and NSF grant DMS 8504322.
} 
In Section 3, we consider the subset intersection graph $G_{n}$. $G_{n}$ has $2^{n}$ vertices, one for each subset of $\{1, \ldots, n\}$, with vertices adjacent if the corresponding sets intersect. We show $(\sqrt{n}+1) / 2 \leq i\left(G_{n}\right) \leq n / 2$. We also consider another graph parameter for this graph. The boxicity of $G$, denoted $b(G)$, is the minimum number of interval graphs whose intersection (as sets of edges) is $G$. Equivalently, it is the minimum $d$ such that $G$ is the intersection graph of $d$-dimensional boxes (products of intervals in each of $d$ coordinates). As with interval number, the boxicity of a graph is as large as the boxicity of any induced subgraph. We show that the boxicity of $G_{n}$ is much higher than its interval number. In particular, when $n$ is even the subgraph of $G_{n}$ induced by the subsets with at most $n / 2$ elements has boxicity $1 / 2\left(\begin{array}{c}n \\ n / 2\end{array}\right)$. Note that there is no relationship between boxicity and interval number; $K_{m, n}$ has boxicity 2 but interval number $\lceil(m n+1) /(m+n)\rceil[8,11]$, while the complement of a complete matching on $n$ vertices has interval number 2 but boxicity $n / 2[7,8]$. Results on both boxicity and interval number, including those used in arguments here, are surveyed in [12].

\section{Representations of cliques}

Most of the results of this section were initially obtained in the thesis of the first author [9]. A surprisingly accurate lower bound comes from a simple counting argument. This basic argument was used independently in [6] for the purpose of bounding the interval number in terms of the clique number.

THEOREM 1. $i_{r}\left(K_{n}\right) \geq\left\lceil 1 / 2\left(\frac{n-1}{r-1}+\frac{r}{n}\right)\right\rceil$.

Proof. Count the edges representable by a $t$-interval representation of depth $r$. Reading from left to right, the initial endpoint of each interval can introduce at most $r-1$ new edges, except that for the first $r-1$ intervals we have a deficiency of $\sum_{i=1}^{r-1} i=\left(\begin{array}{c}r \\ 2\end{array}\right)$. To represent $K_{n}$, we must have $\left(\begin{array}{c}n \\ 2\end{array}\right) \leq n t(r-1)-\left(\begin{array}{c}r \\ 2\end{array}\right)$, or $t \geq 1 / 2\left(\frac{n-1}{r-1}+\frac{r}{n}\right)$.

When $r=2$, this bound reduces to $\lceil n / 2\rceil$, which is achievable, since it is well-known that the edges of $K_{n}$ can be decomposed into $\lceil n / 2\rceil$ paths. For $n>r>2 n+1 / 2-\sqrt{3 n^{2}-n}$, the bound reduces to 2, which is achievable when $r \geq 3 n / 8$. To see this, we use the ad hoc representation in Fig. 1 to show $i_{3}\left(K_{8}\right)=2$. Then $i_{3 p}\left(K_{8 p}\right)=2$ by expanding the set assigned to each vertex into identical sets for $p$ vertices; if $n<8 r / 3$, simply delete some of the intervals that were generated. (Note that there is a "redundancy" in Fig. 1 in that intervals for vertices 4 and 5 meet twice. The effect of forbidding redundancy is discussed in [10].)

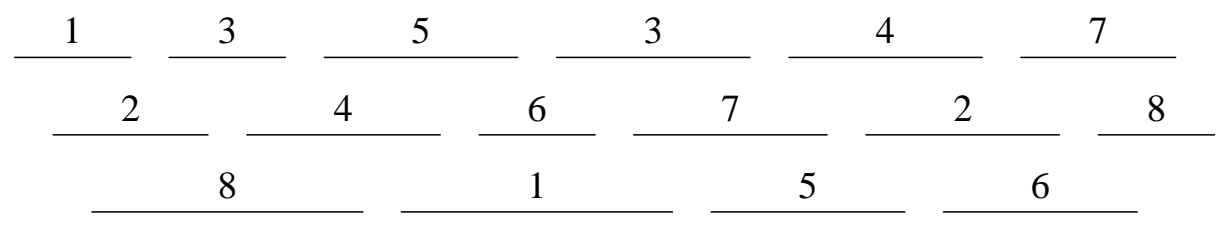

Fig. 1

For arbitrary fixed values of $r$, we have a recursive upper bound construction.

THEOREM 2. $i_{r}\left(K_{n}\right) \leq i_{r}\left(K_{\lceil n / r\rceil}\right)+\lceil 1 / 2(\lceil n / r\rceil+1)\rceil$.

Proof. Partition the vertices of $K_{n}$ into $r$ sets of size at most $\lceil n / r\rceil$. Form a depth $r$ representation for each of the induced cliques. The remaining edges form a complete $r$-partite graph $H$. Since $H$ has no $r+1$-clique, $i_{r}(H)=i(H)$. As obtained in any of $[4,5,7] i(H) \leq\lceil 1 / 2(\lceil n / r\rceil+1)\rceil$. Putting these representations together yields the desired bound.

If $n$ is a power of $r$, we can eliminate the ceiling functions and expand the recurrence to get a closed form bound. We get $i_{r}\left(K_{n}\right) \leq \Sigma_{i=1}^{\log _{r} n} 1 / 2\left(\frac{n}{r^{i}}+1\right) \leq 1 / 2 \frac{n-1}{r-1}+c \log _{r} n$, where $c=1$ if $r$ is even and $c=1 / 2$ if $r$ is odd. To 
get a similar closed-form upper bound in general, we use a slightly weaker recursive bound.

THEOREM 3. $i_{r}\left(K_{n}\right) \leq \frac{n}{2(r-1)}+2\left\lceil\log _{r} n\right\rceil+1$.

Proof. As in the previous proof, partition the vertices of $K_{n}$, but use $r$ parts with exactly $\lfloor n / r\rfloor$ vertices and one part $S$ with $|S|<r$. As argued previously, the subgraph induced by vertices outside $S$ has a depth $r$ representation with $t=i_{r}\left(K_{\lfloor n / r\rfloor}\right)+\lceil 1 / 2(\lfloor n / r\rfloor+1)\rceil$. We can handle the edges involving the remaining vertices by piling up one interval for each, and then placing a small interval for each of the other vertices in the intersection. Since there were less than $r$ leftover vertices, there is a level of depth available for this. This gives the recursive bound $i_{r}\left(K_{n}\right) \leq i_{r}\left(K_{\lfloor n / r}\right)+\lceil 1 / 2(\lfloor n / r\rfloor+1)\rceil+1$.

Fixing $r$ and letting $f(n)=i_{r}\left(K_{n}\right)$, we have $f(n) \leq f(n / r)+\lceil 1 / 2(1+n / r)\rceil+1 \leq f(n / r)+n / 2 r+2$. Iterating $\left\lceil\log _{r} n\right\rceil$ times yields $f(n) \leq\left(n / 2 r+n / 2 r^{2}+\cdots\right)+2\left\lceil\log _{r} n\right\rceil+1 \leq \frac{n}{2(r-1)}+2\left\lceil\log _{r} n\right\rceil+1$.

For all $n$, Theorems 1 and 3 show that $i_{r}\left(K_{n}\right)$ is asymptotic to $n /(2 r-2)$ for fixed $r$. Although they have the same asymptotic behavior, neither of the bounds in Theorems 1 and 2 is exact. Theorem 2 gives $i_{3}\left(K_{8}\right) \leq 3$, but we saw earlier that $i_{3}\left(K_{8}\right)=2$. For an example where the lower bound of Theorem 1 is not tight, consider $i_{6}\left(K_{19}\right)$, where the computation yields $i_{6}\left(K_{19}\right) \geq\lceil 186 / 97\rceil=2$. To show $i_{6}\left(K_{19}\right) \neq 2$, we need an extension of Helly's Theorem.

Helly [3] proved that a finite family of pairwise intersecting intervals has a common point, or in other words that a 1-interval representation of $K_{n}$ has depth at least $n$. Gýarfas and Lehel [2] extended this to consider pairwise intersecting sets that are each composed of more than one interval. If each set is composed of at most 2 intervals, they proved there always exist three points such that every set contains at least one of the three points. Consequently, every 2-interval representation of $K_{n}$ has depth at least $n / 3$. Hence every 2-interval representation of $K_{19}$ has depth at least 7 , so $i_{6}\left(K_{19}\right)>2$. (More generally, they showed there exists a finite number $L(t)$ such that, for every finite collection $F$ of pairwise intersecting sets consisting of at most $t$ intervals each, there is a set of $L(t)$ points meeting each set in $F$. Beyond $L(1)=1$ and $L(2)=3$, no values or reasonable bounds are known.)

\section{The subset intersection graph}

Now consider the graph $G_{n}$ defined in the introduction; the intersection graph of the subsets of an $n$-set. Suppose the elements of the $n$-set are labeled $\{1, \ldots, n\}$.

THEOREM 4. $(\sqrt{n}+1) / 2 \leq i\left(G_{n}\right) \leq n / 2$.

Proof. For the upper bound, there is a simple construction. Assign the subset $A$ intervals $(i-1 / 2, i+1 / 2]$ for each $i \in A$, which lets subsets intersect if and only if they are assigned intersecting intervals. The number of intervals assigned to a set is at most its cardinality. However, if $i, i+1 \in A$, then the intervals $(i-1 / 2, i+1 / 2]$ and $\left(i+1 / 2, i+\frac{3}{2}\right.$ ] form one long interval. Hence the number of intervals assigned to $A$ is actually the number of runs of consecutive elements in $A$, which is at most $n / 2$.

For the lower bound, place the elements in a $\sqrt{n}$ by $\sqrt{n}$ square, and consider the sets formed by the rows and by the columns. Let $H_{n}$ be the subgraph induced by these $2 \sqrt{n}$ sets; we have $i\left(G_{n}\right) \geq i\left(H_{n}\right)$. Since $H_{n}$ is simply $K_{\sqrt{n}, \sqrt{n}}$, it is well-known that $i\left(H_{n}\right)=\lceil(\sqrt{n}+1) / 2\rceil[11]$.

Although $G_{n}$ has many copies of $H_{n}$, the gap between the upper and lower bound here cannot be closed solely by considering these, because the upper bound construct shows that the subgraph induced by all sets of size $\sqrt{n}$ has interval number at most $\sqrt{n}$.

To discuss boxicity, let us consider the subgraph $G_{n}^{\prime}$ induced by the sets of size at most $n / 2$. Of course $b\left(G_{n}\right) \geq b\left(G_{n}^{\prime}\right)$, but we can also give a construction to achieve the lower bound on $b\left(G_{n}^{\prime}\right)$ that does not accomodate addition of the sets of larger size. 
THEOREM 5. If $n$ is even, the $b\left(G_{n}^{\prime}\right)=1 / 2\left(\begin{array}{c}n \\ n / 2\end{array}\right)$.

Proof. The boxicity of a complete multipartite graph is the number of non-trivial parts, as proved in [8]. The subgraph of $G_{n}^{\prime}$ induced by the sets of size $n / 2$ form such a subgraph, with $1 / 2\left(\begin{array}{c}n \\ n / 2\end{array}\right)$ parts, which yields the lower bound.

For the upper bound, we provide a dimension for each complementary pair of sets of size $n / 2$. For the $i$ th complementary pair, let the two sets be $S_{i}$ and $\bar{S}_{i}$. Now, consider an arbitrary set $A$. In the $i$ th coordinate, assign $A$ the interval [0,0], [1,1], or [0,1] according to whether $A \subseteq S_{i}, A \subseteq \bar{S}_{i}$, or $A \cap S_{i} \neq \varnothing \neq A \cap \bar{S}_{i}$. If $A$ and $B$ intersect, then for each dimension there at least one of $S_{i}$ and $\bar{S}_{i}$ meets them both in an element of $A \cap B$. If $A$ and $B$ are dis-

joint, then since $G_{n}^{\prime}$ uses only sets of size at most $n / 2$ there is an $i$ such that $A \subseteq S_{i}$ and $B \subseteq \bar{S}_{i}$ (or vice versa), so that the $i$ th dimension will keep the boxes assigned to $A$ and $B$ from intersecting.

\section{References}

[1] J.R. Griggs and D.B. West, Extremal values of the interval number of a graph, SIAM J. Alg. Disc. Meth. 1(1980), 1-7.

[2] A. Gýarf́as and J. Lehel, A Helly-type problem in trees, in Combinatorial Theory and Its Applications (Erd̈os et al, eds.). North-Holland (1970), 571-584. MR45 \#7602

[3] E. Helly,.-Uber Mengen Kurper mit gemeinschaftlichen Punkten, J. Deutsch Math. Verein 32(1923), 175-176.

[4] L.B. Hopkins and W.T. Trotter, Jr., A bound on the interval number of a complete multipartite graph, in The Theory and Applications of Graphs (Chartrand et al, eds.). Proc. 4th Intl. Conf. Graph Th. (Kalamazoo). Wiley (1981), 391-407. MR83i:05042

[5] L.B. Hopkins, W.T. Trotter, Jr., and D.B. West, The interval number of a complete multipartite graph, Discrete Appl. Math. 8(1984), 163-187.

[6] C. Maas, Some results about the interval number of a graph, Discrete Appl. Math. 6(1983), 99-102.

[7] T.M. Matthews and W.T. Trotter, Jr., The interval number of the complete multipartite graphs. Presented at 2nd Annual SE SIAM Meeting, 1978.

[8] F.S. Roberts, On the boxicity and cubicity of a graph, in Recent Progress in Combinatorics. (W.T. Tutte, ed.). Academic Press (1969).

[9] E.R. Scheinerman, Intersection classes and multiple intersection parameters of graphs. Ph.D. Thesis, Princeton University (1984).

[10] E.R. Scheinerman, Non-redundant interval representations of graphs, to appear.

[11] W.T. Trotter, Jr., and F. Harary, On double and multiple interval graphs. J. Graph Theory 2(1978), 137-142.

[12] D.B. West, Parameters of partial orders and graphs: packing, covering, and representation, in Graphs and Order (I. Rival, ed.). Proc. NATO Adv. St. Inst. (Banff, 1984) D. Reidel (1985), 267-350. 